\title{
Reconhecimento de Palavras, Fluência e Compreensão de Leitura em Alunos com Transtorno do Espectro Autista ${ }^{1}$ Word ReCOGnition, Fluence and Reading Comprehension in Students WITH AUTISM SPECTRUM DISORDER
}

\author{
Camila Fragoso RIBEIRO ${ }^{2}$ \\ Tatiana Pontrelli MECCA ${ }^{3}$ \\ Gabriel Rodriguez BRITO ${ }^{3}$ \\ Alessandra Gotuzo SEABRA ${ }^{4}$
}

\begin{abstract}
RESUMO: Tendo em vista que o desempenho acadêmico é um dos fatores associados ao bom prognóstico no Transtorno do Espectro Autista (TEA), este estudo avaliou diferentes aspectos cognitivos da leitura em oito meninos com TEA, entre 10 e 13 anos $(M=10,87 ; \mathrm{DP}=1,12)$, de escolas públicas e privadas. Avaliou-se o reconhecimento de palavras (TCLPP II), a fluência (TFL) e a compreensão de leitura (TCCL), bem como o Quociente de Inteligência (QI) Estimado (Vocabulário e Raciocínio Matricial do WASI). Os resultados demonstram frequência maior de desempenhos abaixo da média em compreensão de leitura e maior frequência de desempenhos na média tanto no reconhecimento de palavras quanto na fluência de leitura. Em relação ao tempo nas tarefas de fluência e compreensão, houve maior frequência de classificaçóes na média. Não houve correlação significativa entre Vocabulário do WASI e o QI com os testes de leitura. Entretanto, houve correlaçáo positiva, significativa e de alta magnitude entre reconhecimento de palavras com os escores em fluência e compreensão. Uma tendência de magnitude moderada foi observada entre essas duas últimas variáveis. Conhecer o perfil de leitura do aluno com TEA, a partir da avaliaçáo do reconhecimento de palavras, da fluência e da compreensão, possibilita orientar adaptaçôes escolares, com impacto no seu desenvolvimento acadêmico e prognóstico.
\end{abstract}

PALAVRAS-CHAVE: Autismo. Leitura. Compreensão. Vocabulário.

ABSTRACT: Considering that academic performance is one of the factors associated with a good prognosis in the Autism Spectrum Disorder (ASD), this study evaluated different cognitive aspects of reading in 8 boys with ASD, between 10 and 13 years old $(M=10.87 ; S D=1.12)$, of public and private schools. Word recognition (TCLPP II), fluency (TFL) and reading comprehension (TCCL) were evaluated, as well as Estimated IQ (Vocabulary and Matrix Reasoning of the WASI). The results demonstrate a higher frequency of below-average performances in reading comprehension skills, higher frequency of performances on average in word recognition and reading fluency. Regarding the time on fluency and comprehension tasks, there was a higher frequency of average classifications. There was no significant correlation between the Vocabulary and the IQ with the reading tests. However, there was a positive, significant and high magnitude correlation between word recognition with fluency and comprehension scores. A trend of moderate magnitude was observed between these last two variables. The knowledge about the reading profile of students with ASD obtained from the assessment of word recognition, fluency and comprehension makes it possible to guide school adaptations, with an impact on their academic development and prognosis.

KEYWORDS: Autism. Reading. Comprehension. Vocabulary.

\footnotetext{
${ }^{1}$ https://doi.org/10.1590/1980-54702021v27e0050

${ }^{2}$ Graduanda em Psicologia. Bolsista do Programa Institucional de Bolsas de Iniciação Científica (PIBIC) - MackPesquisa. Universidade Presbiteriana Mackenzie. São Paulo/SP/Brasil. E-mail: camimomila@gmail.com. ORCID: https://orcid.org/0000-00024504-5742

${ }^{3}$ Docente. Departamento de Saúde Mental. Programa de Pós-Graduação em Saúde da Comunicação Humana. Faculdade de Ciências Médicas da Santa Casa de São Paulo. São Paulo/SP/Brasil. E-mail: tatiana.mecca@fcmsantacasasp.edu.br. ORCID: https:// orcid.org/0000-0002-2009-6228

${ }^{3}$ Doutorando em Distúrbios do Desenvolvimento. Universidade Presbiteriana Mackenzie. São Paulo/SP/Brasil. E-mail: gabrielrodriguezbrito@gmail.com. ORCID: https://orcid.org/0000-0002-7097-9344

${ }^{4}$ Docente do Programa de Pós-Graduação em Distúrbios do Desenvolvimento. Universidade Presbiteriana Mackenzie. São Paulo/ SP/Brasil. E-mail: alessandra.seabra@makenzie.br. ORCID: https://orcid.org/0000-0002-8373-7897
} 


\section{INTRODUÇÁo}

O diagnóstico de Transtorno do Espectro Autista (TEA) tem sido cada vez mais frequente nos últimos anos e realizado, principalmente, durante a primeira infância (Maenner et al., 2020), de modo que tem crescido o interesse dos pesquisadores sobre aspectos da aprendizagem acadêmica e do manejo comportamental dessa populaçáo na escola. O contexto escolar representa muitos desafios para as crianças com TEA, e o primeiro deles é poder identificar quais as melhores adaptaçôes e acomodaçóes que podem favorecer a sua aprendizagem. Nesse sentido, a avaliação de habilidades acadêmicas pode contribuir para o estabelecimento de diretrizes que permitirão o desenvolvimento do aluno com TEA no ambiente escolar (Antunes et al., 2020). Uma das habilidades que deve ser investigada em seus diferentes domínios é a leitura.

A competência da leitura influencia o desenvolvimento social e individual, com impacto no acesso a informaçóes, conhecimentos e nos níveis de participaçáo e inclusão na sociedade (Krug, 2015; Seabra \& Capovilla, 2010). No Brasil, o Indicador de Alfabetismo Funcional (Inaf) estima que três a cada dez brasileiros náo dominam plenamente as habilidades de leitura, escrita e matemática (INAF, 2018). As dificuldades na aprendizagem da leitura e da escrita aparecem já nos primeiros anos escolares, atingindo de 30\% a $40 \%$ das crianças brasileiras (Andrade et al., 2014). Estas podem ser mais frequentes em alunos com TEA, devido ao impacto que as alteraçóes cognitivas, linguísticas e de comunicação social possuem sobre a leitura (Arvigo et al., 2018; Brown et al., 2013; Carnahan et al., 2011; Mayes \& Calhoun, 2008).

Alguns dos aspectos que se relacionam com a leitura de palavras e a compreensão leitora dos indivíduos com TEA são o funcionamento intelectual geral (Mayes \& Calhoun, 2008) e o déficit de coerência central. Este se refere a uma falha em integrar detalhes e informaçóes em apenas um conceito ou uma ideia (Booth \& Happé, 2010), o que afeta o desenvolvimento espontâneo de habilidades metacognitivas de leitura que assistem a compreensão textual (Nunes \& Walter, 2016). Déficits de Teoria da Mente (Wellman, 2018) também podem afetar a compreensáo de textos, na medida em que dificultam o entendimento da motivaçáo e do estado emocional dos personagens, limitando os alunos a uma leitura e a um entendimento literal do conteúdo (Carnahan et al., 2011).

Ainda em relação à cognição, os déficits executivos observados no TEA, como organização, planejamento, monitoramento e iniciação de comportamentos para atingir objetivos (Demetriou et al., 2018), também se apresentam como obstáculo para a compreensão leitora. Déficits nas funçôes executivas interferem nos processos de organização de informaçóes, como sequenciar os eventos em uma história; no planejamento, como acessar conhecimentos prévios e realizar conexóes entre as informaçôes; e no monitoramento da sua própria compreensão leitora, como, por exemplo, construir imagens mentais, engajar-se em discussóes e resumir o que foi lido (Nguyen et al., 2015).

Além disso, considera-se a importância do processamento visual para o reconhecimento da palavra escrita, pois permite a identificação de uma letra, independentemente da posição que ela ocupe na palavra, no tamanho ou no formato. Essa invariância perceptual possibilita o reconhecimento de pequenas diferenças entre palavras com um conjunto semelhante de letras em termos de posiçẫo e formato (Dehaene, 2013). Outrossim, uma das habilidades que mais impactam a aquisição e o desenvolvimento da leitura é a linguagem oral (Davidson et al., 2018). 
Crianças com TEA que apresentam atrasos na aquisição e no uso de suas competências linguísticas podem apresentar dificuldades em compreender instruçóes e responder às demandas escolares que são, em sua maioria, pautadas em instruçóes verbais (Volkmar \& Wiesner, 2019). Há indicadores de alteraçôes variadas nos diversos aspectos da linguagem, como na Fonologia, na Semântica, na Sintaxe e na Pragmática. O desempenho acadêmico, portanto, será afetado de forma e em níveis variados, em função do tipo e da gravidade do prejuízo linguístico (Brignell et al., 2018; Brown et al., 2013).

Prejuízos nos aspectos pragmáticos podem impactar a realização de inferências durante a leitura e a compreensão de regras e de comportamentos que devem ser adotados em situações que demandam atividades de leitura. Alunos com TEA apresentam maiores dificuldades na leitura de textos sociais quando comparados a textos não sociais, e a compreensão do material lido está mais associado ao nível da linguagem oral do que ao diagnóstico de TEA (Brown et al., 2013; Volkmar \& Wiesner, 2019).

Além da linguagem oral, o reconhecimento de palavras é uma das variáveis que impactam a compreensão de leitura de crianças com TEA (Davidson et al., 2018). Estudos prévios apontam que o modelo de compreensão de leitura descrito por Gough e Tunmer (1986) é o resultado da compreensão linguística somada ao reconhecimento de palavras, o qual também se observa em crianças com TEA (Brown et al., 2013; Davidson et al., 2018; McIntyre et al., 2017).

De modo geral, o reconhecimento de palavras encontra-se relativamente preservado em pessoas com TEA; quando prejuízos estão presentes, estes estão associados a atrasos na linguagem oral (Davidson et al., 2018). Outros aspectos também têm sido descritos como fatores que contribuem para a compreensão de leitura nos indivíduos com TEA, como os aspectos semânticos e morfossintáticos da linguagem (Brown et al., 2013; Cronin, 2014).

Segundo Dias et al. (2014), remediar as dificuldades de leitura implica entender a interação entre os diversos processos cognitivos e as habilidades que culminam nessa complexa competência. De acordo com o Modelo Componencial da Leitura, o desempenho em leitura envolve três domínios: cognitivo, psicológico e ecológico. O campo cognitivo é composto pelo reconhecimento de palavras, pela fluência e pela compreensão oral, fundamentais para a compreensão leitora, que é a habilidade complexa de extrair significado a partir do texto escrito (Joshi \& Aaron, 2012). O reconhecimento de palavras é a habilidade de decodificação pela rota fonológica e de acesso ao léxico pela rota lexical. A compreensão oral refere-se a uma habilidade mais ampla e anterior à linguagem escrita propriamente dita, que faz referência à compreensão da linguagem de forma geral. Já a fluência refere-se à velocidade de processamento. Mesmo com uma decodificação precisa, um leitor poderá ter dificuldades na compreensão de leitura caso a velocidade seja muito baixa (Dias et al., 2014; Jacobson et al., 2011).

Diversos estudos sobre leitura e TEA descrevem habilidades de reconhecimento de palavras e de compreensão de leitura (Brown et al. 2013; Davidson et al., 2018; McIntyre et al. 2017), mas com pouca ênfase nas habilidades de fluência. No entanto, há evidências de que a fluência também é um importante preditor de compreensão de leitura, com uma contribuição única, que vai além do reconhecimento de palavras (Solari et al., 2017). Desse modo, o objetivo deste estudo foi descrever os padróes de leitura de crianças diagnosticadas com TEA a partir dos componentes de reconhecimento de palavras, compreensão de leitura e fluência, 
bem como verificar a relação entre os diferentes componentes com vocabulário (como medida de compreensão oral) e inteligência geral.

\section{Método}

Trata-se de um estudo descritivo, transversal e correlacional, o qual descreve e analisa o perfil de crianças com TEA, nos diferentes componentes cognitivos da leitura, em um momento específico, e relaciona tais características com outras variáveis, como vocabulário e inteligência.

\subsection{Participantes}

Participaram do estudo oito crianças do sexo masculino diagnosticadas com TEA por uma equipe multidisciplinar, com base nos critérios do Manual Diagnóstico e Estatístico de Transtornos Mentais (DSM-5) da Associação Americana de Psiquiatria - APA (2014). Os participantes foram selecionados a partir dos prontuários de duas instituiçôes que atuam no diagnóstico e/ou no atendimento de crianças com TEA na cidade de São Paulo. Foram incluídos pacientes com fala preservada que compreendem e respondem às instruçóes verbais, alfabetizados e que cursam o Ensino Fundamental em escolas regulares. Não foram incluídos participantes com deficiência intelectual (Quociente de Inteligência - QI $\leq 70$ ) ou que apresentavam comorbidades genéticas, neurológicas ou com outros transtornos do neurodesenvolvimento. $\mathrm{O}$ Quadro 1 sumariza as características dos participantes.

\section{Quadro 1}

Descrição dos participantes com TEA e características em termos de idade, ano escolar, tipo de escola, QI e vocabulário

\begin{tabular}{|c|c|c|c|l|l|}
\hline Participante & Idade & Ano & \multicolumn{1}{|c|}{ Escola } & \multicolumn{1}{|c|}{ QI } & \multicolumn{1}{|c|}{ Vocabulário } \\
\hline A & 10 & $4^{\circ}$ & Particular & 78 (limítrofe) & 30 (muito inferior) \\
\hline B & 10 & $4^{\circ}$ & Particular & 109 (média) & 50 (média) \\
\hline C & 10 & $4^{\circ}$ & Particular & 110 (média) & 59 (média) \\
\hline D & 10 & $4^{\circ}$ & Particular & 84 (abaixo da média) & 30 (muito inferior) \\
\hline E & 11 & $5^{\circ}$ & Particular & 84 (abaixo da média) & 53 (média) \\
\hline F & 11 & $5^{\circ}$ & Pública & 77 (limítrofe) & 30 (muito inferior) \\
\hline G & 12 & $6^{\circ}$ & Particular & 103 (média) & 45 (média) \\
\hline H & 13 & $7^{\circ}$ & Pública & 83 (abaixo da média) & 38 (inferior) \\
\hline
\end{tabular}

\subsection{INSTRUMENTOS}

Como instrumentos desta pesquisa, foram utilizados:

- Teste de Competência de Leitura de Palavras e Pseudopalavras para o Ensino FundamentalII - TCLPP-II (Brito, 2017), o qual avalia o reconhecimento de palavras e de pseudopalavras de estudantes do $4^{\circ}$ ao $9^{\circ}$ ano. É composto por 40 palavras e 60 pseudopalavras (criadas com base em palavras reais, mas com alteração em ao menos uma letra). O objetivo é assinalar um "X" na coluna ao lado da lista de palavras, indicando se a palavra está 
escrita de forma correta ou errada. São cinco tipos de itens: Corretas Regulares (a relação letra/som é biunívoca: ovada); Corretas Irregulares (palavras ortograficamente corretas e irregulares na leitura e na escrita (por exemplo, anexar); Trocas Visuais (palavras ortograficamente incorretas, com inversão, substituição ou trocas visuais de letras com grafia similar ("mairdo" por "marido"); Trocas Fonológicas (palavras ortograficamente incorretas, com trocas fonológicas ("físiga” por "física”); e Trocas Ortográficas (palavras ortograficamente incorretas, porém homófonas (“mássimo” por "máximo"). Para garantir a compreensão, foram ministrados cinco itens de treino, não considerados para a análise. A pontuação consiste em acertos (um ponto) e erros (zero ponto) e foi usada como medida de reconhecimento de palavras.

- $\quad$ Teste de Fluência de Leitura - TFL (Brito, 2017), o qual avalia a fluência de estudantes do $4^{\circ}$ ao $9^{\circ}$ ano. Ele possui duas partes: (P1) uma ficha de leitura com 150 palavras isoladas que variam de acordo com as características psicolinguísticas de frequência (alta, média e baixa) e a regularidade (regular e irregular); e (P2) que é composta por um texto narrativo com 452 palavras. A tarefa proposta é ler corretamente, sem que se altere a entonação e a pronúncia correta, no menor tempo possível, embora seja dito ao examinando que não há um tempo limite. Foram aplicados dez itens de treino. Foram analisadas a quantidade de palavras lidas corretamente e o tempo total na tarefa como medidas de fluência.

- $\quad$ Teste Cloze de Compreensão de Leitura - TCCL (Brito, 2017), o qual avalia a compreensão de leitura de estudantes do $4^{\circ}$ ao $9^{\circ}$ ano. É dividido em duas partes: (P1) texto narrativo (240 palavras e 60 itens); e (P2) texto dissertativo/expositivo (172 palavras e 43 itens). Trata-se de Teste Cloze de múltipla escolha, no qual há omissão sistemática de palavras no texto. A omissão é feita a cada quinto vocábulo, (independentemente de classe gramatical ou significado). A palavra omitida é substituída por um traço. Na parte inferior de cada traço, há uma caixa com quatro alternativas de palavras, sendo uma delas o vocábulo correto, um vocábulo de sentido aproximado e dois vocábulos distratores, apresentados em ordem aleatória. A tarefa é assinalar qual das quatro alternativas é a que melhor completa a lacuna. O primeiro parágrafo de cada texto foi aplicado como treino. Os escores nos dois textos foram usados como medidas de compreensão de leitura.

- Subtestes Vocabulário e Raciocínio Matricial da Escala de Inteligência Wechsler Abreviada - WASI (versão brasileira adaptada por Trentini et al., 2014), os quais avaliam a inteligência de indivíduos entre 6 e 89 anos. Foram utilizados os subtestes de Vocabulário e de Raciocínio Matricial para estimar o QI. No Vocabulário, a criança explica o significado de determinada palavra e, no Raciocínio Matricial, o objetivo é escolher, entre cincos opçóes, aquela que completa uma matriz corretamente. Os escores foram convertidos em pontos ponderados e somados para calcular o QI estimado. A pontuação em escore $\mathrm{T}$ do Vocabulário foi usada como medida de compreensão oral.

\subsection{Procedimentos}

Este estudo é parte de uma pesquisa mais ampla de desenvolvimento e de estudos de validade de instrumentos, submetida e aprovada pelo Comitê de Ética em Pesquisa (CEP). Após o consentimento das instituições, os responsáveis legais das crianças foram contatados 
e convidados a dar consentimento para a coleta de dados. As aplicaçóes dos testes ocorreram nas instituiçôes, em sessóes únicas, com duração aproximada de 1 hora e 30 minutos. Os responsáveis assinaram o Termo de Consentimento Livre e Esclarecido (TCLE), e os próprios participantes consentiram verbalmente em participar da pesquisa.

\subsection{ANÁLISE DE DADOS}

Para a série de casos, foi conduzida uma análise descritiva do desempenho individual em cada teste. As medidas do TCCL, TFL e TCLPP-II foram comparadas aos resultados descritos por Brito (2017) com crianças com desenvolvimento típico. No TCCL e no TLF, foram avaliadas a acurácia da leitura dos textos e das palavras, bem como o tempo de execução. No TCLPP-II foi considerada a medida de acurácia (acerto e erro).

Os resultados dos participantes foram classificados como: "médio" (entre -1 e +1 , desvio-padrão da média dos participantes com desenvolvimento típico); “inferior” (entre -2 e -1, desvios-padrão da média); "muito inferior" (abaixo de -2, desvios-padrão da média); "superior" (entre +1 e +2 , desvios-padrão da média); e "muito superior" (acima de +2 , desvios-padrão da média). Para a classificação do desempenho, foram utilizados dados de média e desvio-padrão retirados de Brito (2017). Ressalta-se que um participante era do $5^{\circ}$ ano do Ensino Fundamental de escola pública, e em função da ausência de estudos prévios com alunos com desenvolvimento típico referente a esse ano escolar, os desempenhos desse participante foram comparados com os de crianças do $6^{\circ}$ ano do Ensino Fundamental de escola pública.

O desempenho dos participantes no subteste Vocabulário do WASI foi comparado com as amostras de normatização do teste correspondente à idade. Foi considerada a medida ponderada do escore de acertos - escore $\mathrm{T}$ ( média $=50$, desvio-padrão $=10$ ).

Ademais, foi realizada uma síntese de frequência dos participantes nos diferentes níveis de classificação e uma descrição do perfil de cada participante nos diferentes instrumentos. Dessa forma, foi possível analisar o perfil dos participantes tanto com um enfoque nomotético, ao comparar o desempenho de cada participante a um referencial normativo, quanto com um enfoque ideográfico, no qual a identificação de possíveis prejuízos é concebida a partir de modelos de processamento de informação de análise intraindivíduo (Haase et al., 2018). Por fim, em função do número reduzido de participantes, foi conduzida a análise não paramétrica de correlação de Spearman entre os testes de leitura, o subteste Vocabulário do WASI e o QI Estimado. Foi utilizado o software IBM SPSS, versão 21.0. O nível de significância adotado foi de 5\%.

\section{Resultados e discussáo}

A Tabela 1 sumariza os desempenhos dos participantes no TCLPP II, que avalia o reconhecimento de palavras, em comparação com estudantes sem transtorno de neurodesenvolvimento. Foi considerado como medida o escore total de palavras indicadas corretamente. Observa-se que cinco participantes se classificaram como desempenho médio; dois participantes classificaram-se como desempenho inferior; e um participante classificou-se como desempenho muito inferior. 


\section{Tabela 1}

Resultados do desempenho dos participantes com TEA no TCLPP II em comparação com os participantes de desenvolvimento típico

\begin{tabular}{c|c|c|c|l}
\hline Participante & Escore & Média de referência & DP de referência & Classificaçáo \\
\hline A & 69 & 84 & 11 & Inferior \\
\hline B & 60 & 84 & 11 & Muito inferior \\
\hline C & 87 & 84 & 11 & Média \\
\hline D & 79 & 84 & 11 & Média \\
\hline E & 92 & 85 & 9 & Média \\
\hline$F^{*}$ & 58 & 77 & 15 & Inferior \\
\hline G & 93 & 87 & 8 & Média \\
\hline H & 64 & 78 & 21 & Média \\
\hline
\end{tabular}

Nota: Desempenho comparado com estudantes de $6^{\circ}$ ano de escolas públicas. DP = Desvio Padrão.

O Quadro 2 refere-se aos desempenhos e aos tempos de execução (em minutos) dos participantes no subteste Palavras do TFL, em comparação a estudantes de neurodesenvolvimento típico. As medidas consideradas foram o escore total de palavras lidas corretamente e o tempo de execução da tarefa (em minutos). Como é possível observar, três participantes tiveram ambos desempenho e tempo de execução na média; um participante teve o desempenho inferior e o tempo de execução muito inferior; um participante teve o desempenho inferior e o tempo de execução médio; um participante teve desempenho muito superior e tempo de execução médio; um participante teve desempenho médio e tempo de execução muito inferior; e um participante teve desempenho médio e tempo de execução superior.

\section{Quadro 2}

Resultados do desempenho e do tempo de execução (em minutos) dos participantes com TEA no subteste Palavras do TFL em comparação com os participantes de desenvolvimento típico

\begin{tabular}{|c|c|c|c|c|c|}
\hline Participante & Medida & Tempo /Escore & Média de referência & DP de referência & Classificaçáo \\
\hline \multirow{2}{*}{ A } & Tempo & $7 \min 56 s$ & $4 \min 47 \mathrm{~s}$ & $1 \min 4 s$ & Muito inferior \\
\hline & Escore & 115 & 131 & 10,6 & Inferior \\
\hline \multirow{2}{*}{ B } & Tempo & $4 \min 28 s$ & $4 \min 47 s$ & $1 \mathrm{~min} 4 \mathrm{~s}$ & Média \\
\hline & Escore & 118 & 131 & 10,6 & Inferior \\
\hline \multirow{2}{*}{$\mathrm{C}$} & Tempo & $4 \min 42 s$ & $4 \min 47 s$ & $1 \mathrm{~min} 4 \mathrm{~s}$ & Média \\
\hline & Escore & 137 & 131 & 10,6 & Média \\
\hline \multirow{2}{*}{$\mathrm{D}$} & Tempo & $5 \min 20 s$ & $4 \min 47 s$ & $1 \min 4 s$ & Média \\
\hline & Escore & 128 & 131 & 10,6 & Média \\
\hline \multirow{2}{*}{$\mathrm{E}$} & Tempo & $3 \min 11 s$ & $3 \min 49 s$ & $1 \mathrm{~min} 6 \mathrm{~s}$ & Média \\
\hline & Escore & 144 & 131 & 6 & Muito superior \\
\hline
\end{tabular}




\begin{tabular}{|c|c|c|c|c|l|}
\hline \multirow{2}{*}{$\mathrm{F}^{*}$} & Tempo & $6 \min 57 \mathrm{~s}$ & $3 \min 56 \mathrm{~s}$ & $1 \min 20 \mathrm{~s}$ & Muito inferior \\
\cline { 2 - 6 } & Escore & 102 & 120 & 20 & Média \\
\hline \multirow{3}{*}{$\mathrm{G}$} & & & & $52 \mathrm{~s}$ & Superior \\
\hline & Tempo & $2 \min 15 \mathrm{~s}$ & $3 \min 41 \mathrm{~s}$ & 12 & Média \\
\cline { 2 - 6 } & Escore & 139 & 133 & & \\
\hline \multirow{3}{*}{$\mathrm{H}$} & & & & $1 \mathrm{~min} 10 \mathrm{~s}$ & Média \\
\cline { 2 - 6 } & Tempo & $4 \min 10 \mathrm{~s}$ & $3 \min 13 \mathrm{~s}$ & 19 & Média \\
\cline { 2 - 6 }
\end{tabular}

Nota: Desempenho comparado com estudantes de $6^{\circ}$ ano de escolas públicas. DP = Desvio Padrão.

O Quadro 3 sumariza os resultados dos participantes no TCCL, o qual avalia a compreensão de leitura e a compara com a média de estudantes sem transtornos do neurodesenvolvimento. Foram consideradas as medidas de acerto (escores) e de tempo (em minutos) nos textos narrativos e dissertativos e no escore total em ambos os textos. Como pode ser observado, três participantes tiveram desempenhos na média em todas as medidas; dois tiveram desempenhos mistos entre inferiores e médios; dois tiveram desempenhos mistos entre inferior e muito inferior; e um teve desempenho muito inferior em todas as medidas. Verifica-se que cinco participantes tiveram tempo de execução na média em todas as medidas; dois tiveram tempos de execução mistos entre superiores (foram mais rápidos) e médios; e um teve tempo de execução misto entre inferior e médio.

\section{Quadro 3}

Desempenho e tempo (em minutos) dos participantes com TEA no TCCL em comparação com os participantes de desenvolvimento tipico

\begin{tabular}{|c|c|c|c|c|c|c|c|}
\hline $\begin{array}{c}\text { Partici- } \\
\text { pante }\end{array}$ & Texto & Escore & $\begin{array}{l}M(D P) \text { de } \\
\text { referência }\end{array}$ & Classificaçáo & Tempo & $\begin{array}{l}M(D P) \text { de } \\
\text { referência }\end{array}$ & Classificaçáo \\
\hline \multirow{3}{*}{ A } & Narrativo & 18 & $43( \pm 8)$ & Muito inferior & 7 & $14( \pm 4)$ & Superior \\
\hline & Dissertativo & 7 & $27( \pm 7)$ & Muito inferior & 8 & $10( \pm 3)$ & Média \\
\hline & Total & 25 & $70( \pm 14)$ & Muito inferior & & & \\
\hline \multirow{3}{*}{ B } & Narrativo & 20 & $42(+8)$ & Inferior & 12 & $141+1)$ & Módi \\
\hline & Dissertativo & 12 & $27( \pm 7)$ & Muito inferior & 5 & $10( \pm 3)$ & Superior \\
\hline & Total & 41 & $70( \pm 14)$ & Muito inferior & & & \\
\hline \multirow{3}{*}{$\mathrm{C}$} & Narrativo & 28 & $43( \pm 8)$ & Inferior & 14 & $14( \pm 4)$ & Média \\
\hline & Dissertativo & 24 & $27( \pm 7)$ & Média & 9 & $10( \pm 3)$ & Média \\
\hline & Total & 52 & $70( \pm 14)$ & Inferior & & & \\
\hline \multirow{3}{*}{$\mathrm{D}$} & Narrativo & 34 & $43( \pm 8)$ & Inferior & 16 & $14( \pm 4)$ & Média \\
\hline & Dissertativo & 12 & $27( \pm 7)$ & Muito inferior & 8 & $10( \pm 3)$ & Média \\
\hline & Total & 46 & $70( \pm 14)$ & Inferior & & & \\
\hline \multirow{3}{*}{ E } & $\begin{array}{l}\text { Narrativo } \\
\end{array}$ & 47 & $45( \pm 9)$ & Média & 23 & $16( \pm 8)$ & Média \\
\hline & Dissertativo & 25 & $29( \pm 6)$ & Média & 16 & $11( \pm 4)$ & Inferior \\
\hline & Total & 72 & $74( \pm 12)$ & Média & & & \\
\hline \multirow{3}{*}{$\mathrm{F}$} & Narrativo & 22 & $37( \pm 12)$ & Inferior & 16 & $18( \pm 5)$ & Média \\
\hline & Dissertativo & 16 & $22( \pm 8)$ & Média & 7 & $10( \pm 4)$ & Média \\
\hline & Total & 38 & $58( \pm 19)$ & Inferior & & & \\
\hline
\end{tabular}




\begin{tabular}{|l|l|c|c|l|c|c|l|}
\hline \multirow{4}{*}{$G$} & Narrativo & 49 & $49( \pm 5)$ & Média & 13 & $17( \pm 6)$ & Média \\
\cline { 2 - 8 } & Dissertativo & 38 & $32( \pm 7)$ & Média & 8 & $9( \pm 3)$ & Média \\
\cline { 2 - 8 } & Total & 87 & $81( \pm 11)$ & Média & & & \\
\hline \multirow{3}{*}{$\mathrm{H}$} & & & & & & & \\
\cline { 2 - 8 } & Narrativo & 35 & $43( \pm 10)$ & Média & 23 & $22( \pm 7)$ & Média \\
\cline { 2 - 8 } & Dissertativo & 22 & $22( \pm 8)$ & Média & 12 & $11( \pm 4)$ & Média \\
\cline { 2 - 8 } & Total & 57 & $64( \pm 18)$ & Média & & & \\
\hline
\end{tabular}

Nota: Desempenho comparado com estudantes de $6^{\circ}$ ano de escolas públicas. $\mathrm{M}=$ Média; DP = Desvio Padrão.

A Tabela 2 sintetiza a frequência das classificaçóes dos participantes quando comparadas aos estudantes sem transtorno de neurodesenvolvimento. Foram consideradas todas as medidas de desempenho e de tempo de execução de cada um dos testes (TCLL, TFL, TCLPP II e Compreensão Oral). No TCCL, constata-se que, no escore narrativo, um participante se classificou como muito interior, quatro como inferior e três na média; no escore dissertativo, três participantes classificaram-se como muito inferior e cinco na média; no escore total, dois participantes classificaram-se como muito inferior, três como inferior e três na média; no tempo narrativo, sete participantes classificaram-se na média e um como muito superior; e no tempo dissertativo, um participante classificou-se como inferior, sete na média e um como superior. Já no TFL, observa-se que, no escore texto, um participante se classificou como muito inferior, três como inferior, três como na média e um como superior; no escore palavras, dois classificaram-se como inferior, cinco na média e um como superior; e no tempo palavras, dois participantes classificaram-se como muito inferior, cinco como na média e um como superior. No TCLPP II, verifica-se que, no escore total, um participante se classificou como muito inferior, dois como inferior e cinco como na média. Por fim, no WASI, três participantes classificaram-se como muito inferior, um como inferior e quatro como na média.

\section{Tabela 2}

Frequência dos participantes, nas faixas de classificação, em comparação com os participantes de desenvolvimento tipico, nas habilidades avaliadas

\begin{tabular}{l|c|c|c|c|c}
\hline \multicolumn{1}{c|}{ Medidas } & \multicolumn{5}{c}{ Classificaçáo } \\
\hline \multicolumn{1}{c|}{ Compreensáo (TCCL) } & Muito inferior & Inferior & Média & Superior & Muito superior \\
\hline Escore narrativo & 1 & 4 & 3 & - & - \\
\hline Escore dissertativo & 3 & - & 5 & - & - \\
\hline Escore total & 2 & 3 & 3 & - & - \\
\hline Tempo narrativo & - & - & 7 & 1 & - \\
\hline Tempo dissertativo & - & 1 & 6 & 1 & - \\
\hline \multicolumn{1}{c|}{ Fluência (TFL) } & Muito inferior & Inferior & Média & Superior & Muito superior \\
\hline Escore Texto & 1 & 3 & 3 & 1 & - \\
\hline Escore Palavras & - & 2 & 5 & - & 1 \\
\hline Tempo Palavras & 2 & - & 5 & 1 & - \\
\hline & & & & & \\
\hline
\end{tabular}




\begin{tabular}{c|c|c|c|c|c}
\hline $\begin{array}{c}\text { Reconhecimento de } \\
\text { palavras (TCLPP II) }\end{array}$ & Muito inferior & Inferior & Média & Superior & Muito superior \\
\hline Escore Total & 1 & 2 & 5 & - & - \\
\hline & & & & & \\
\hline $\begin{array}{c}\text { Compreensão Oral } \\
\text { (Vocabulário - WASI) }\end{array}$ & Muito inferior & Inferior & Média & Superior & Muito superior \\
\hline Escore Ponderado & 3 & 1 & 4 & - & - \\
\hline
\end{tabular}

Após as descrições realizadas em função dos desempenhos em cada instrumento, foi feita uma descrição dos perfis de cada um dos participantes em todos os testes aplicados. $\mathrm{O}$ Quadro 4, a seguir, sumariza o desempenho de cada participante nas quatro habilidades avaliadas. Foram consideradas todas as medidas do TCCL, do TCLPP II, do TFL e do subteste Vocabulário do WASI.

\section{Quadro 4}

Desempenho geral de cada participante em cada uma das habilidades avaliadas

\begin{tabular}{|c|c|c|c|c|c|c|c|c|}
\hline \multirow{3}{*}{ Medidas } & \multicolumn{8}{|c|}{ Classificaçóes } \\
\hline & \multicolumn{4}{|c|}{$4^{\circ}$} & \multicolumn{2}{|c|}{$5^{\circ}$} & \multirow{2}{*}{$\begin{array}{l}6^{0} \\
G\end{array}$} & \multirow{2}{*}{$\begin{array}{l}7^{\mathbf{o}} \\
\mathrm{H}\end{array}$} \\
\hline & $\mathbf{A}$ & B & C & D & $\mathbf{E}$ & $\mathbf{F}^{*}$ & & \\
\hline $\begin{array}{l}\text { Escore Narrativo } \\
\text { (TCCL) }\end{array}$ & $\begin{array}{l}\text { Muito } \\
\text { inferior }\end{array}$ & Inferior & Inferior & Inferior & Média & Inferior & Média & Média \\
\hline $\begin{array}{l}\text { Escore Dissertativo } \\
\text { (TCCL) }\end{array}$ & $\begin{array}{l}\text { Muito } \\
\text { inferior }\end{array}$ & $\begin{array}{l}\text { Muito } \\
\text { inferior }\end{array}$ & Média & $\begin{array}{l}\text { Muito } \\
\text { inferior }\end{array}$ & Média & Média & Média & Média \\
\hline Escore Total (TCCL) & $\begin{array}{l}\text { Muito } \\
\text { inferior }\end{array}$ & $\begin{array}{l}\text { Muito } \\
\text { inferior }\end{array}$ & Inferior & Inferior & Média & Inferior & Média & Média \\
\hline $\begin{array}{l}\text { Tempo Narrativo } \\
\text { (TCCL) }\end{array}$ & Superior & Média & Média & Média & Média & Média & Média & Média \\
\hline $\begin{array}{l}\text { Tempo Dissertativo } \\
\text { (TCCL) }\end{array}$ & Média & Superior & Média & Média & Inferior & Média & Média & Média \\
\hline Escore Texto (TFL) & Inferior & Inferior & Média & Inferior & Média & $\begin{array}{l}\text { Muito } \\
\text { inferior }\end{array}$ & Superior & Média \\
\hline Escore Palavras (TFL) & Inferior & Inferior & Média & Média & $\begin{array}{l}\text { Muito } \\
\text { superior }\end{array}$ & Média & Média & Média \\
\hline Tempo Palavras (TFL) & $\begin{array}{l}\text { Muito } \\
\text { inferior }\end{array}$ & Média & Média & Média & Média & $\begin{array}{l}\text { Muito } \\
\text { inferior }\end{array}$ & Superior & Média \\
\hline $\begin{array}{l}\text { Escore Total } \\
\text { (TCLPP-II) }\end{array}$ & Inferior & $\begin{array}{l}\text { Muito } \\
\text { Inferior }\end{array}$ & Média & Média & Média & Inferior & Média & Média \\
\hline Vocabulário (WASI) & $\begin{array}{l}\text { Muito } \\
\text { inferior }\end{array}$ & Média & Média & $\begin{array}{l}\text { Muito } \\
\text { inferior }\end{array}$ & Média & $\begin{array}{l}\text { Muito } \\
\text { inferior }\end{array}$ & Média & Inferior \\
\hline
\end{tabular}

Nota: Desempenho comparado com estudantes de $6^{\circ}$ ano de escolas públicas. 
O participante $\mathrm{A}$ apresentou desempenho abaixo do esperado em todas medidas. Já em relação às medidas de tempo no TCCL, A obteve classificação média e superior, diferente do observado no TLF, no qual obteve classificação muito inferior no tempo de leitura. O participante $B$ apresentou semelhanças em relação ao participante $\mathrm{A}$, com desempenho inferior nas medidas de leitura, porém com tempo de execução adequado. Apenas no vocabulário seu desempenho foi na média esperada para a idade. $\mathrm{O}$ participante $\mathrm{C}$ apresentou desempenho abaixo do esperado apenas na acurácia do texto narrativo do TCCL, com impacto no escore total do TCCL, indicando uma dificuldade mais específica com esse tipo de texto. $\mathrm{O}$ participante $\mathrm{D}$ apresentou dificuldades tanto de vocabulário, quanto de compreensão e fluência de leitura de textos. Entretanto, apresentou classificação média em relação ao reconhecimento e à leitura de palavras, bem como ao tempo de execução das tarefas. $\mathrm{O}$ participante $\mathrm{E}$ apresentou desempenho na média na maioria das medidas, com execução de uma dificuldade no tempo de execução do texto dissertativo. Já o participante $\mathrm{F}$ apresentou desempenho bastante variado, com dificuldades de acurácia no texto narrativo e no escore total do TCCL, na fluência da leitura de textos e no tempo de leitura de palavras; ademais, foram observadas dificuldades no reconhecimento de palavras (TCLPP-II) e no vocabulário. Por fim, os participantes $\mathrm{G}$ e H apresentaram bons desempenhos, tanto em relação à acurácia quanto em relação ao tempo de execução.

Por um lado, as análises de correlações de Spearman indicaram que não houve correlação significativa entre o subteste de Vocabulário do WASI com o TCLPP-II (rho=0,46; $\mathrm{p}=0,247$ ), com o TFL ( $r h o=0,66 ; \mathrm{p}=0,076$ ) e com o TCCL ( $r h o=0,56 ; \mathrm{p}=0,148$ ). Também não foi observada correção significativa entre o QI Estimado com o TCLPP-II (rho=0,48; $\mathrm{p}=0,230$ ), com o TFL ( $r h o=0,66 ; \mathrm{p}=0,076$ ) e com o TCCL ( $r h o=0,43 ; \mathrm{p}=0,272$ ). Por outro lado, houve correlação positiva, significativa e de alta magnitude entre reconhecimento de palavras (TCLPP-II) com os escores em TFL ( $r h o=0,90 ; p=0,002)$ e TCCL ( $r h o=0,74 ; p=0,037$ ). Uma tendência de magnitude moderada foi observada entre os escores totais no TFL e no TCCL (rho=0,69; p=0,058).

Considerando que o desempenho acadêmico é um dos fatores que contribuem para um melhor prognóstico nos indivíduos com TEA (Klein, 2009), este estudo teve por objetivo descrever o desempenho em leitura de oito meninos com TEA, sem deficiência intelectual associada. A avaliação de leitura foi realizada considerando os componentes do domínio cognitivo, conforme o modelo de Joshi e Aaron (2012), a saber: o reconhecimento de palavras, a fluência, a compreensão oral e a compreensão de leitura. Para tal, foram utilizados instrumentos desenvolvidos por Brito (2017), com dados prévios de validade e de normatização para estudantes com desenvolvimento típico do $6^{\circ}$ ao $9^{\circ}$ ano do Ensino Fundamental, bem como subtestes do WASI.

Como é possível observar, nas medidas de reconhecimento de texto e de fluência, houve uma predominância de classificaçôes "média”, tendo uma minoria de participantes com desempenho inferior ou superior em comparação aos alunos sem transtorno de desenvolvimento. Já nas medidas que avaliaram a compreensão de texto, houve uma distribuição mais acentuada de classificaçóes "inferior" e "muito inferior" no escores, e nenhum dos participantes teve desempenho superior ou muito superior nos escores em compreensão de leitura. Já os tempos de execução da tarefa se mantiveram dentro da média. Esses resultados corroboram estudos prévios que relatam dificuldade de compreensão leitora em indivíduos com TEA (Brown et al., 2013; Davidson et al., 2018; Gomes \& Souza, 2016; Nunes \& Walter, 2016). 
Apesar do desempenho de cinco participantes constarem como "muito inferior" ou "inferior" no escore geral do TCCL, destaca-se uma pequena discrepância entre os subtestes. No escore dissertativo, a maioria dos participantes concentra-se na "média", enquanto no escore narrativo, a maior distribuição ocorreu nas classificações abaixo da média. A literatura prévia aponta que existe uma relação entre compreensão e gêneros textuais e destaca que textos do gênero narrativo tendem a demandar mais inferências contextuais sobre os estados dos personagens (Spinillo \& Almeida, 2014). Desse modo, uma possível hipótese explicativa para tal resultado é que, além das habilidades de linguagem oral (Davidson et al., 2018), dificuldades no desenvolvimento da Teoria da Mente também poderiam comprometer a compreensão de leitura em indivíduos com TEA, na medida em que dificulta o entendimento da motivação e do estado emocional dos personagens, limitando os alunos a uma leitura e a um entendimento literal do conteúdo (Carnahan et al., 2011; Nunes \& Walter, 2016). Crianças com TEA apresentaram desempenho significativamente inferior na compreensão e na interpretação de histórias em que precisavam inferir estados mentais dos personagens (Velloso et al., 2013). Por isso, estudos futuros podem contribuir para a compreensão do efeito de determinados gêneros textuais na compreensão de pessoas com TEA.

Ao analisar-se o desempenho individual de cada participante, verificaram-se diversas dissociaçóes entre as habilidades avaliadas. McIntyre et al. (2017) apontam três padróes diferentes de leitores com TEA de alto funcionamento (sem déficit intelectual), a depender do comprometimento dos sintomas: 1) leitores com problemas de compreensão, mas com funcionamento esperado quando comparado ao seu grupo de referência em leitura oral de palavras (medida que avalia reconhecimento de palavras e fluência simultaneamente); 2) leitores com problemas globais: déficits nas medidas de reconhecimento, leitura de palavras e compreensão; e 3) leitores medianos: performance esperada ou superior nas habilidades de leitura. Nota-se que os participantes C, D e F apresentam desempenho semelhante ao descrito no primeiro caso. Os desempenhos nas provas de leitura dos participantes A e B apresentam características semelhantes ao segundo grupo, enquanto os participantes E, G e $\mathrm{H}$ apresentam características mais compatíveis com o terceiro quadro. Esses achados colaboram para a heterogeneidade de perfil presente nos indivíduos com TEA (Brignell et al., 2018; Brown et al., 2013; McIntyre et al., 2017).

Observou-se também que apenas alunos do $4^{\circ}$ ano do Ensino Fundamental se encaixaram no primeiro perfil, com prejuízos mais específicos de compreensão de leitura. Isso pode indicar o desenvolvimento de habilidades compensatórias para lidar com as dificuldades de leitura nos anos mais avançados, uma vez que a presença de estratégias compensatórias no TEA está relacionada, entre outros fatores, com o grau de escolaridade (Livingston, 2020). Além disso, Davidson et al. (2018) verificaram que a idade, as habilidades de compreensão oral e a gravidade do quadro de TEA são fatores que contribuem para os déficits na compreensão leitora.

Outrossim, constatou-se que, para os participantes A, D e F, houve uma concomitância de desempenhos inferiores nas medidas de compreensão de leitura e de compreensão oral, enquanto os participantes $\mathrm{B}, \mathrm{C}$ e $\mathrm{H}$ apresentaram uma dissociação dessas habilidades. No caso dos participantes B e C, é possível levantar a hipótese de que outros prejuízos em habilidades distintas à compreensão oral podem impactar a compreensão de leitura, tais como as funçóes executivas, a Teoria da Mente e o nível geral de funcionamento intelectual (Carnahan et al., 
2011; Demetriou et al., 2018; Mayes \& Calhoun, 2008; Nunes \& Walter, 2016). Ademais, a divergência desses resultados talvez resida no fato de que ter o diagnóstico de TEA é um preditor forte para o desempenho prejudicado em compreensão de leitura (Davidson et al., 2018). No entanto, essa hipótese não é endossada por outros autores (Brown et al., 2013; Volkmar \& Wiesner, 2019), pois sugerem que os prejuízos de leitura nos indivíduos com TEA não se devem ao diagnóstico em si, mas ao nível de comprometimento na linguagem oral. Já no caso do participante $\mathrm{H}$, é possível considerar o uso de estratégias compensatórias, conforme descrito por Livingston (2020).

Especificamente sobre as medidas de fluência, Solari et al. (2017) observaram que a fluência de leitura tem um papel significativo para a compreensão leitora de pessoas com TEA. O mesmo estudo apontou que alunos com TEA tendem a ter um desempenho igual aos pares sem transtorno de desenvolvimento na leitura de palavras isoladas, mas desempenho prejudicado na leitura oral de textos. Essa perspectiva pode ser observada no desempenho dos participantes D, E, F nos diferentes subtestes do TFL, visto que apenas o participante G apresentou um desempenho diferente do perfil descrito por Solari et al. (2017), com um melhor desempenho na leitura de texto do que na leitura de palavras.

Desse modo, apesar de um certo grau de variância nas habilidades de leituras comprometidas, o estudo de McIntyre et al. (2017) averiguou que todos os perfis de padrão de leitura apresentavam dificuldades em realizar inferências e integrar informaçôes. Essas descobertas apoiam a explicação de que outras características associadas ao TEA podem contribuir para as dificuldades de compreensão leitora, tais como a disfunção executiva, ou melhor, a dificuldade da população com TEA em executar de tarefas complexas e simultâneas, característica que pode impactar não só o desempenho em compreensão, mas, também, as medidas mais complexas de fluência, como as de textos (Mcintyre et al., 2017; Nguyen et al., 2015; Solari et al., 2017).

Como limitações deste estudo, destaca-se o número reduzido de participantes em função das restrições decorrentes das medidas de isolamento social impostas pela pandemia do SARS-CoV-2. Além disso, dentro do modelo componencial de leitura, o aspecto da compreensão auditiva foi pouco explorado, uma vez que outras ferramentas poderiam fornecer mais informaçóes a respeito da compreensão auditiva sem a demanda da expressão verbal, como é necessário para responder ao subteste Vocabulário. Ademais, não foi investigada a relação entre leitura e outras variáveis, como as funçóes executivas, a Teoria da Mente, a Coerência Central e o grau de comprometimento de acordo com os sintomas apresentados pelos participantes. Desse modo, sugere-se que estudos futuros explorem mais sobre os perfis de leitura dos alunos com TEA e as hipóteses causais subjacentes, a fim de elaborar intervençóes e adaptaçóes curriculares que promovam uma maior inclusão e qualidade de vida para alunos com o diagnóstico de TEA.

\section{Conclusốes}

A partir da descrição do perfil de desempenho em testes de leitura, observou-se a ocorrência de padróes de reconhecimento de palavras, fluência e compreensão leitora em crianças com diagnóstico de TEA. Verificou-se nos participantes deste estudo que a habilidade de compreensão tende a estar mais deficitária que o reconhecimento de palavras e a fluência. 
Notou-se ainda que, possivelmente, as dificuldades de compreensão dependem dos gêneros textuais. Maiores dificuldades foram observadas na compreensão de texto narrativo.

Os resultados também indicaram que, embora o desempenho nos testes tenha se mostrado heterogêneo, uma característica bastante comum nos indivíduos com TEA, houve uma relação positiva, significativa e de alta magnitude entre o reconhecimento de palavras com os demais componentes, a fluência e a compreensão.

\section{REFERÊNCIAS}

Andrade, E. M. D. A., Mecca, T. P., Almeida, R. P. D., \& Macedo, E. C. D. (2014). Eficácia de um programa de intervenção fônica para crianças com dificuldades de leitura e escrita. Revista Psicopedagogia, 31(95), 119-129.

Antunes, A. M., Mecca, T. P., \& Júlio-Costa, A. (2020). Transtorno do Espectro do Autismo: avaliação neuropsicológica, intervençôes e consideraçôes sobre inclusão escolar. In R. P. Fonseca, A. G. Seabra, \& M. C. Miranda (Orgs.), Neuropsicologia escolar (1 ${ }^{a}$ ed., pp. 453-492). Pearson.

Associação Americana de Psiquiatria. (2014). Manual Diagnóstico e Estatístico de Transtornos Mentais $\left(\mathrm{DSM}-5^{\oplus}\right)$. Artmed.

Arvigo, M. C., Saad, A. G., Signorelli, F., Haddad., R. G. C., \& D’Antino, M. E. F. (2018). Transtorno do Espectro do Autismo e Comunicação. In C. A. de la H. Amato., D. Brunoni, \& P. S. Boggio (Orgs.), Distúrbios do desenvolvimento: estudos interdisciplinares (1a ed., pp. 455-470). Memnon.

Booth, R., \& Happé, F. (2010). "Hunting with a knife and... fork": Examining central coherence in autism, attention deficit/hyperactivity disorder, and typical development with a linguistic task. Journal of Experimental Child Psychology, 107(4), 377-393. https://doi.org/10.1016/j. jecp.2010.06.003

Brignell, A., Morgan, A. T., Woolfenden, S., Klopper, F., May, T., Sarkozy, V., \& Williams, K. (2018). A systematic review and meta-analysis of the prognosis of language outcomes for individuals with autism spectrum disorder. Autism \& Developmental Language Impairments, 3, 1-19. https://doi. org/10.1177/2396941518767610

Brito, G. R. (2017). Desenvolvimento e evidências de validade e precisão de instrumentos de avaliação dos componentes de leitura no ensino fundamental II [Dissertação de Mestrado, Universidade Presbiteriana Mackenzie, Faculdade de Distúrbios do Desenvolvimento]. Repositório da Universidade Presbiteriana Mackenzie. http://tede.mackenzie.br/jspui/bitstream/tede/3238/5/ Gabriel\%20Rodriguez\%20Brito.pdf

Brown, H. M., Oram-Cardy, J., \& Johnson, A. (2013). A meta-analysis of the reading comprehension skills of individuals on the autism spectrum. Journal of Autism and Developmental Disorders, 43(4), 932-955. https://doi.org/10.1007/s10803-012-1638-1

Carnahan, C. R., Williamson, P. S., \& Christman, J. (2011). Linking cognition and literacy in students with autism spectrum disorder. Teaching Exceptional Children, 43(6), 54-62. https://doi. org/10.1177/004005991104300606

Cronin, K. (2014). The relationship among oral language, decoding skills, and reading comprehension in children with autism. Exceptionality, 22(3), 141-157. https://doi. org/10.1177/004005991104300606 
Davidson, M. M., Kaushanskaya, M., \& Weismer, S. E. (2018). Reading comprehension in children with and without ASD: The role of word reading, oral language, and working memory. Journal of Autism and Developmental Disorders, 48(10), 3524-3541. https://doi.org/10.1007/s10803-018-3617-7

Dehaene, S. (2013). A aprendizagem da leitura modifica as redes corticais da visão e da linguagem verbal. Letras de Hoje, 48(1), 148-152. https://revistaseletronicas.pucrs.br/ojs/index.php/fale/ article/view/12113/8892

Demetriou, E. A., Lampit, A., Quintana, D. S., Naismith, S. L., Song, Y. J. C., Pye, J. E., Hickie, I., \& Guastella, A. J. (2018). Autism spectrum disorders: a meta-analysis of executive function. Molecular psychiatry, 23(5), 1198-1204. https://doi.org/10.1038/mp.2017.75

Dias, N. M., Seabra, A. G., \& Montiel, J. M. (2014). Instrumentos de avaliação de componentes da leitura: investigação de seus parâmetros psicométricos. Avaliação Psicológica, 13(2), 235-245.

Gomes, C. G. S., \& Souza, D. D. G. D. (2016). Ensino de sílabas simples, leitura combinatória e leitura com compreensão para aprendizes com autismo. Revista Brasileira de Educação Especial, 22(2), 233252. https://doi.org/10.1590/S1413-65382216000200007

Gough, P., \& Tunmer, W. (1986). Decoding, reading, and reading disability. Remedial and Special Education, 7(1), 6-10. https://doi.org/10.1177/074193258600700104

Haase, V. G., Gauer, G., \& Gomes, C. M. A. (2018). Neuropsicometria: modelos nomotético e ideográfico. In L. F. Malloy-Diniz, D. Fuentes, P. Mattos, \& N. Abreu (Orgs.), Avaliação Neuropsicológica (1ª ed., pp. 22-30). Artmed.

Indicador de Alfabetismo Funcional. (2018). Indicador de Alfabetismo Funcional: INAF BRASIL 2018 Resultados Preliminares. https://drive.google.com/file/d/1ez-6jrlrRRUm9JJ3MkwxEUffltCTEI6/view.

Jacobson, L. A., Ryan, M., Martin, R. B., Ewen, J., Mostofsky, S. H., Denckla, M. B., \& Mahone, E. M. (2011). Working memory influences processing speed and reading fluency in ADHD. Child Neuropsychology, 17(3), 209-224. https://doi.org/10.1080/09297049.2010.532204

Joshi, R. M., \& Aaron, P. G. (2012). Componential Model of Reading (CMR) validation studies. Journal of Learning Disabilities, 45(5), 387-390. https://doi.org/10.1177/0022219411431240

Klein, S. K. (2009). Avaliação neuropsicológica: conceitos básicos e utilidade clínica. In R. Tuchman, \& I. Rapin (Orgs.), Autismo: Abordagem neurobiológica. (1ª ed., pp. 284-300). Artmed.

Krug, F. S. (2015). A importância da leitura na formação do leitor. Revista de Educação do IDEAU, 22(10), 1-13.

Livingston, L. A., Shah, P., Milner, V., \& Happé, F. (2020). Quantifying compensatory strategies in adults with and without diagnosed autism. Molecular autism, 11(15), 1-10. https://doi.org/10.1186/ s13229-019-0308-y

Maenner, M. J., Shaw, K. A., Baio. J., Washington, A., Patrick, M., DiRienzo, M., Christensen, D. L., Wiggins, L. D., Pettygrove, S., Andrews, J. G., Lopez, M., Hudson, A., Baroud, T., Schwenk, Y., White, T., Rosenberg, C. R., Lee, L.-C., Harrington, R. A., Huston, M., ... Dietz, P. M. (2020). Prevalence of Autism Spectrum Disorder Among Children Aged 8 Years - Autism and Developmental Disabilities Monitoring Network, 11 Sites, United States, 2016. MMWR Surveill Summ, 69(4), 1-12. http://dx.doi.org/10.15585/mmwr.ss6904a1

Mayes, S. D., \& Calhoun, S. L. (2008). WISC-IV and WIAT-II profiles in children with highfunctioning autism. Journal of autism and developmental disorders, 38(3), 428-439. https://doi. org/10.1007/s10803-007-0410-4 
McIntyre, N. S., Solari, E. J., Grimm, R. P., Lerro, L. E., Gonzales, J. E., \& Mundy, P. C. (2017). A comprehensive examination of reading heterogeneity in students with high functioning autism: Distinct reading profiles and their relation to autism symptom severity. Journal of Autism and Developmental Disorders, 47(4), 1086-1101. https://doi.org/10.1007/s10803-017-3029-0

Nguyen, N. N., Leytham, P., Schaefer Whitby, P., \& Gelfer, J. I. (2015). Reading comprehension and autism in the primary general education classroom. The Reading Teacher, 69(1), 71-76. https://doi. org/10.1002/trtr.1367

Nunes, D. R. D. P., \& Walter, E. C. (2016). Literacy process in students with Autism Spectrum Disorder (ASD): a review study. Revista Brasileira de Educação Especial, 22(4), 619-632. https:// doi.org/10.1590/S1413-65382216000400011

Seabra, A. G., \& Capovilla, F. C. (2010). Alfabetização: método fônico (5ª ed.). Memnon.

Solari, E. J., Grimm, R., McIntyre, N. S., Swain-Lerro, L., Zajic, M., \& Mundy, P. C. (2017). The relation between text reading fluency and reading comprehension for students with autism spectrum disorders. Research in Autism Spectrum Disorders, 41, 8-19. https://doi.org/10.1016/j. rasd.2017.07.002

Spinillo, A. G., \& Almeida, D. D. (2014). Compreendendo textos narrativo e argumentativo: há diferenças? Arquivos Brasileiros de Psicologia, 66(3), 115-132.

Trentini, C. M., Yates, D. B., \& Heck,0 V. S. (2014). Manual da adaptação para população brasileira da Escala Wechsler Abreviada de Inteligência - WASI (1 $1^{\text {a }}$ ed.). Pearson.

Velloso, R. D. L., Duarte, C. P., \& Schwartzman, J. S. (2013). Evaluation of the theory of mind in autism spectrum disorders with the Strange Stories Test. Arquivos de Neuro-psiquiatria, 71(11), 871-876. https://doi.org/10.1590/0004-282X20130171

Volkmar, F., \& Wiesner, L. A. (2019). Autismo: guia essencial para compreensão e tratamento. Artmed.

Wellman, H. M. (2018). Theory of mind: The state of the art. European Journal of Developmental Psychology, 15, 728-755. https://doi.org/10.1080/17405629.2018.1435413-

Recebido em: 24/03/2021

Reformulado em: 13/07/2021

Aprovado em: 20/07/2021 